\title{
STRUKTUR UND PERSONAL Der ReICHSJUGendFÜHRUNG, 1941 - 1945
}

Der Reichsleiter für die

Jugenderziehung der NSDAP

Baldur von Schirach

Reichsjugendführer der NSDAP und

Jugendführer des Deutschen Reichs

Adjutant

Persönl. Referent

Artur Axmann

Bf. Gerhard Weltzin

Stabsführer ${ }^{2}$

Bf. Dr. Gerhard Hüring

Adjutant

Persönl. Referent

Helmut Möckel

Stf. Gerhard Freese

Hbf. Hans Franke

BDM-Reichsreferentin

Persönl. Mitarbeiterin

Beauftragte für das BDM-Werk "Glaube und Schönheit"

Dr. Jutta Rüdiger

Ogf. ${ }^{3}$ Marianne Leipold, ab 1/194l Ogf. Hedy Böhmer

Ogf. Martha Stöckl,

davor bis 5/1941 Ogf. Annemarie Kaspar, ab 2/1942 Jutta Rüdiger

\section{Unmittelbare Stellen bzw. Hauptabteilungen außerhalb der Ämter}

\section{Zentralamt ${ }^{4}$}

HAL

HAL

AL

AL

AL

AL

AL

AL

AL

AL

AL

\section{Behördenabteilung 5}

HAL

\section{Kriegsbetreuungsdienst}

HAL

AR

$A L$

AL ab 1940 Gbf. Friedrich Knoop, danach Ogbf. Herbert Moiller ab 1942 Gbf. Friedrich Knoop, ab 11/1943 Hbf. Hans Franke

Bf. Felix Trautmann

Obf. Kurt Brasche

Bf. Dr. Paul Blümel

Hbf. Harald Döscher

Hmf. Ottilie Habermehl

Obf. Heinrich Hoffmann

Obf. Gustav Höpken

Bf. Dr. Gerhard Hüring

Bf. Dr. Werner Kugler

Bf. Dr. Werner Schmidt

Ostf. Hans Wimmer

Gbf. Heinrich Liler, ab 8/1941 Hbf. Otto-Heinrich Braun

Obf. Heinrich Schulz

Ogbf. Herbert Müller, Hbf. Dr. Hansgeorg Moka

Obf. Helmut Blum

ab 11/1942 Gmf. Eva Todtenhöfer

Bf. Andreas Leidenberger

Hmf. Lotte Meier

1 Im Erlaß Hitlers vom 2.8.1940 wurde Schirach - neben der Anweisung zur Beibehaltung des Amtes des Reichsleiters für die Jugenderziehung der NSDAP - außerdem zum "Beauftragten des Führers für die Inspektion der gesamten Hitler-Jugend auch im staatlichen Bereich" emannt; mit dieser im Dritten Reich nur selten gebrauchten Amtsbezeichnung sollten auch Kontrollmöglichkeiten für den Sektor staatlicher Jugendpolitik geschaffen werden.

2 Der Stabsführer der Hitler-Jugend war nominell gleichzeitig Stellvertreter des Reichsjugendführers, und wurde während dessen Abwesenheit - z.B. bei Fronteinsätzen - jeweils zum "Bevollmächtigten Vertreter des Jugendführers des Deutschen Reichs und des Reichsjugendfiihrers der NSDAP" emannt.

3 Die Dienstbezeichnung "Obergauführerin" (Ogf.) ist mit Wirkung vom 1.12.1941 offiziell abgeschafft und durch "Gebietsmädelführerin" (Gmf.) ersetzt worden; diese Regelung wurde jedoch nicht durchgängig beachtet.

4 In das Zentralamt wurde im Januar 1943 im Zuge "kriegsbedingter Kräfteeinsparungen" die Behördenabteilung eingegliedert; die Leitung des Zentralamtes übernahm der bisherige Leiter der Behördenabteilung, Hbf. Otto-Heinrich Braun.

5 Die Behördenabteilung ist im Zuge "kriegsbedingter Kräfteeinsparungen" im Januar 1943 aufgelöst und ins Zentralamt eingegliedert worden. 
Dienststelle

Kinderlandverschickung

HAL

AR

AL

AL

AL

$\mathrm{AL}$

AL

AL

AL

AL

$\mathrm{AL}$

AL
Helmut Möckel i.V. von B.v. Schirach, in der RJF für KLV zuständig ab 2/1941 Obf. Heinrich Schulz, ab 6/1942 Hbf. Eberhard Grüttner, ab 9/1944 Obf. Richard Heil

Obf. Gerhard Dabel

Ogf. Marianne Leipold, ab 6/1942 Gmf. Dr. Ursula Kuhlo

Obf. Ganzsprengel

Obf. Josef Loskill

Bf. Hans Hinnerks

Gf. Annermarie Loeschky

Bf. Erich Fehlberg

Bmf. Erika Oldenburg

Hmf. Gretel Seelig

Hbf. Johannes Teetz

Bf. Karl-Heinz Thürmann

Obf. Konrad Uebler

Der Beauftragte für die Leibeserziehung der deutschen Jugend

Ogbf. Hans von Tschammer und Osten

Auslands- und Volkstumsamt (bzw. Auslands- und volkspolitisches Amt) ${ }^{6}$

Hbf. Dr. Walter Schmidt

HAL Volksdeutsche Jugendarbeit

Hbf. Dr. Heinz Schmidt, Hbf. Hans Lauterbacher

Hbf. Emil Teichmann

HAL ab 1943 Dr. Walter Fink, ab 1944 Obf. Herbert Brinkmann

Bf. Erhard Wessely

HAL

Bf. Bernhard Feuerriegel

HAL Jugendarbeit in den besetzten Ostgebieten

Hbf. Siegfried Nickel

HAL Auslandsdeutsche Jugend

AR

Obf. Helmut Antoni

Gmf. Elka von Wedel

$\mathrm{AL}$

Bf. Keith Stobbe

AL

Bf. Heinrich Jürgens

AL

bis $5 / 1941$ Ugf. Hildegard Tapken

AL

Hgf. Ernst Grunow

AL

Gff. Heinz Gollmar

Kommandeur der Adolf-Hitler-Schulen

AL

Gbf. Kurt Budāus, ab 1942 Gbf. Kurt Petter

Obf. Adalbert Garthe

AL

Obf. Hellmut Rinklin

AL

Obf. Valentin Kronberger

Ostf. Wilhelm Schrank

AL

Obf. Walter Garde

\section{Hauptämter und Ämter der Reichsjugendführung}

\section{Hauptamt 1}

Personalamt

HAL (Überwachung)

HAL

HAL

HAL

HAL

HAL (Streifendienst)

AR

AL

$\mathrm{AL}$

AL

$\mathrm{AL}$
Ogbf. Heinz-Hugo John, ab 1/1943 Gbf. Kurt Budäus

Ogbf. Heinz-Hugo John, ab 1/1941 und ab 3/1941 Gbf. Kurt Budăus, ab 3/1941 Hbf. Wilhelm Maus, ab 4/1943 Hbf. Herbert Finkentey, 1942 und ab 1944 Hbf. Willy Lohel

Obf. William Knopp, ab 8/1943 Bf. Siegfried Kulas

Obf. Theodor Rabe

Bf. Friedrich Hengst

Obf. Emst-Berhard Gasch (bis 1943) Obf. Gerhard Ertelt

Ostf. Edmund Heuser

Gmf. Hedy Böhmer

Obf. Albert Hoffmann Bf. Willi Reppert

Ugf. Gertrud Stoffregen Bmf. Gerda Dahl

6 Bis Ende 1941 existierten das Grenz- und volkspolitische Amt unter Leitung von Hbf. Theodor Stadler und das von Bf. Hans Lauterbacher geleitete (Grenz- und) Auslandsamt nebeneinander. Nach der kriegsbedingten Zusammenführung beider Dienststellen im nunmehrigen Grenz- und Auslandsamt ist diese Behörde im Dezember 1941 in Auslands- und Volkstumsamt/Auslands- und Volkspolitisches Amt umbenannt worden; danach lösten sich der neue Amtschef Schmidt und der bisherige Amtsinhaber Lauterbacher bei jeweiligen Militäreinsåtze in der Führung ab. 


\section{Organisationsamt}

HAL

AR $\mathrm{AL}$
Ogbf. Heinz-Hugo John/Gbf. Werner Kley, ab 3/1941 Obf. Gerhard Kalinke, ab 6/194 Gbf. Erich Steinacker, ab 10/1943 Hbf. Karl Jeckstadt-Borchert, ab 1/1944 Hbf. Otto Würschinger Obf. Josef Kremers, ab 1941 Obf. Otto Würschinger bis 1/1941 Ogf. Marianne Leipold, Gmf. Eva Todtenhöfer, ab 11/1942 Gmf. Hedy Böhmer ab 3/1943 Hmf. Sabine Schmidt (bis 10/1943) Obf. Karl Jeckstadt Borchert

\section{Amt HJ-Gerichtsbarkeit}
HAL
HAL
HAL
AR

$\mathrm{AL}$

Mob.-Dienststelle ${ }^{7}$ (Der Mob.-Beauftragte) HAL

Arbeitsausschuß Langemarck
Ogbf. Heinz-Hugo John, ab 4/194I Hbf. Werner Hess, ab 1/1943 Obf. Walter Tetzlaff, ab 12/1943 Hbf. Hans Kleeberg

Obf. Hans Grieshammer Obf. Fritz Nilli

Obf. Walter Tetzlaff Gf. Schulz, ab 3/1944 Hmf. Ilse Pfleumer Hbf. Dr. Adam Möller bis 10/1940 Gbf. Heinrich Lüer, Hbf. Dr. Hansgeorg Moka Obf. Ernst-Bernhard Gasch Ogbf. Heinz-Hugo Johm, ab 1942 Hbf. Dr. Hansgeorg Moka

\section{Hauptamt II}

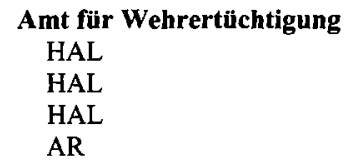

AL für Motor $\mathrm{HJ}$

AL für Nachrichten-HJ

AL für Flieger-HJ

Inspekteur für die Wehrertüchtigungslager

AL

AL

Amt für Leibesübungen

HAL

HAL

AR

$\mathrm{AL}$

AL

AL

AL

AL

Sportauswertung

Amt für Führerausbildung und Schulen ${ }^{8}$ AR
Ogbf. Dr. Ernst Schlünder

Ogbf. Dr. Ernst Schlünder

Obf. Hans Pastor

Obf. Heinz Voigtländer

Bf. Hans-Karl Loewer ab 6/1944 Hmf. Hedwig Stolz

Bf. Walter Borchert

Hgf. Erich Mathes

Hbf. Major Adolf Dickfeld

ab 10/1942 Bf. Gerhard Hein, ab 9/1944 Obf. Erich Bärenfänger

Bf. Werner Paulick

Hgf. Heinz-Ulrich Balz

Ogbf. Dr. Ernst Schlünder, 1940 und ab 1942 Hbf. Gerhard Abelbeck

Obf. Walter Seidemann

Stf. Herbert Lehmann bis 4/1940 Gf. Cläre Schwerdt, Gmf. Ingeborg Wolffgramm, ab 4/1944 Hmf. Barbara Metger

Bf. Wilhelm Wagner

Obf. Hans Franke

Stf. Gerhard Schulze

Stf. Werner Hartmann ab 8/1943 Mhgf. Ellen Aeilts

Gbf. Kurt Budăus Gmf. Mathilde Ochs

\footnotetext{
7 Die Mob-Dienststelle ist im Zuge "kriegsbedingter Kräfteeinsparungen" im Januar 1943 ins Personalamt eingegliedert worden.

8 Das Amt Führerausbildung und Schulen ist im Zuge "kriegsbedingter Kräfteeinsparungen" im Januar 1943 ins Personalamt eingegliedert worden.
} 


\section{Hauptamt III}

Amt für weltanschauliche Schulung
AR
HAL
HAL
HAL
HAL

AL

AL

AL

AL

Kulturamt
HAL
HAL
HAL
HAL
HAL
AR

Presse- und Propaganda-Amt

Geschäftsführerin

HAL

HAL

HAL

Verbindungsführer zum RmfVuP

AR

$\mathrm{AL}$

AL

AL

AL
Gbf. Fritz Brennecke', ab 1942 Hbf. Gottfried Griesmayr Ugf. Anneliese Bartel, Gmf. Mathilde Ochs, ab 1/1942 Hmf. Henriette Wäsche

Hbf. Gerhard Schubert Obf. Paul Gierlichs

Bmf. Liselotte Bastian Hmf. Ilse Bluhm Hbf. Albert Kilian

Bf. Wilhelm Lehmann

Bf. Helmut Andersen

Prof. Dr. Hans Werner Grebe

Ogbf. Dr. Rainer Schlösser, Obf. Siegfried Raeck, ab 1943 Hbf. Otto Zander

Obf. Heinrich Hartmann

Bf. Wolfgang Stumme

Bf. Hermann Roth

Ostf. Helmut Majewski bis 1942 Stf. Günther Boehnert bis 11/1939 Hedwig Banicki, bis 5/1944 Gmf. Magdalene Noelle, ab 8/1944 Hmf. Annemarie Becker
Reichsinstitut für nationalsozialistische Jugendarbeit ${ }^{10}$

HAL $\mathrm{AL}$

Reichsschrifttumstelle ${ }^{11}$ (Reichsjugendbücherei) AL
Hbf. Günter Kaufmann, ab 1940 Hbf. Gustav Memminger

Bmf. Milly Raabe

Bf. Alfred Weidenmann

Ostf. Franz Winterhager

Bf. Gerhard Fröhner

Obf. Günther Dietrich

ab 1 1/1940 Hmf. Ruth Gensicke, ab 1/1944 Mrf. Melita Maschmann

Bf. Erich Witteberg

Hgf. Herbert Schulz

Gustav Schierholz

Stf. Theo Vogel

Hbf. Günter Kaufmann, ab 1940

Bf. Albert Mưller, ab 1942 Ogbf. Dr. Rainer Schlösser

Bf. Albert Müller

Stf. Dr. Georg Ebersbach

Obf. Fritz Helke, ab 9/1942 Hbf. Gustav Memminger

Mrf. Liselotte Bastian

\section{Hauptamt IV}

\section{Soziales Amt}

HAL und Ltr. der Kommunaldienststelle

HAL und stellv. Ltr, des Berufswettkampfes in der DAF

HAL und Ltr. der Rechtsdienststelle

HAL

HAL

HAL

HAL

AR

AL der Rechtsdienststelle
Hbf. Otto Schroeder

Bf. Willi Heidemann, ab 1942 Hbf. Otto Schroeder, (zugleich Leiter der Reichsarbeitsgemeinschaft für Jugendbetreuung)

Hbf. Wolfgang Schlinke

Hbf. Hans Wiese

Obf. Heinrich Boldt, ab 1943 Hgf. Dr Gerhard Klemer (zugleich Geschäftsführer der Reichsarbeitsgemeinschaft für Jugendbetreuung)

Bf. Willi Heidemann

Bf. Walter Bergemann bis $10 / 1943$ Bf. Otto Heuer

Obf. Dr. Kurt Fricke

Gmf. Erna Pranz

Herbert Vornefeld

9 Zwischen 11/1940 und 2/1941 amtierte mit Ogf./Gmf. Mathilde Ochs, bislang BDM-Amtsreferentin des Amtes für weltanschauliche Schulung, erstmals eine Frau als K-Amtschef eines selbständigen Amtes der Reichsjugendführung.

10 Das Reichsinstitut für nationalsozialistische Jugendarbeit ist im Zuge "kriegsbedingter Kräfteeinsparungen" im Januar 1943 ins Presseund Propagandaamt eingegliedert worden.

11 Die Reichsschrifttumsstelle ist im Zuge "kriegsbedingter Kräfteeinsparungen" im Januar 1943 ins Amt für weltanschauliche Schulung eingegliedert worden. 
AL

$\mathrm{AL}$

AL

$\mathrm{AL}$

$\mathrm{AL}$

AL

$\mathrm{AL}$
Ostf. Alwin Brockmann Bf. Leopold Ost Bf. Rolf Ahrens Stf. Kurt Riese Hmf. Gertrud Albrecht Stf. Hermann Trölsch Obf. Heinz Lübke

Ogbf. Dr. Robert Hördemann" ${ }^{12}$ ab 2/1941 Obf. Dr. Richard Liebenow Bf. Dr. Eberhard Kitzing, ab 7/1942 Dr. Walter Neun

Ostf. Dr. Horst Dreyer Ogf. Dr. Ursula Kuhlo, ab 6/1942 Bmf. Dr. Gertrud Huhn

Obf. Dr. Gerhard Joppich Ostf. Dr. Wilhelm Breipohl

Hmf. Dr. Erika Geisler

Mrf. Dr. Eva Domansky Mrf. Dr. Josephine Mayer

Hbf. Rudi Peuckert, ab 2/1942 Obf. Simon Winter

Obf. Ernst Schulz

Bf. Hans Menzel, Bf. Karl Hornung Ogf. Luise Essig, ab 7/1943 Hmf. Gertrud Paust ab $7 / 1943$ Hmf. Frieda Herbold

Obf. Albert Breunsbach Ostf. Otto Stolle

\section{Hauptamt V}

Bauamt

HAL

HAL

HAL

AL

AL

$\mathrm{AL}$

$\mathrm{AL}$

$\mathrm{AL}$

AL
Ogbf. Johannes Wilhelm Rodatz

Hbf. Willy Heitmüller, ab 10/1941 Obf. Gerhard Krause

Bf. Hans-Joachim Leue

Obf. Günther David

Obf. Dr. Theodor Steimle

Gff. Erwin Jahnke Bf. Fritz Abt

Bf. Otto Biedermann

Obf. Heinrich Haack

Bf. Wilhelm Meyer

Bf. Walter Schulenburg
Amt für Heime und Jugendherbergen ${ }^{14}$ AR

Arbeitsausschuß für HJ-Heimbeschaffung

$\mathrm{AL}$

Reichsverband für Deutsche Jugendherbergen
Obf. Günther David, 1942 Hbf. Ernst Langer, ab l 1/1942 Obf. Günther David Gf. Eva Todtenhöfer Hbf. Willy Heitmüller, ab 1942 Obf. Philipp Wohlschläger Ostf. Dr. Theodor Steimle Ogbf. Johannes Wilhelm Rodatz

12 Zwischen 12/1940 und 2/1941 war Ogf. Dr. Ursula Kuhlo kurzzeitig K-Chef[in] des Gesundheitsamtes der RJF.

13 Am 13.3.1940 als Amt Bauerntum und Ostland eingerichtet, im Oktober 1940 umbenannt.

14 Das Amt für Heime und Jugendherbergen ist im Zuge "kriegsbedingter Kräfteeinsparungen" im Januar 1943 in das Bauamt eingegliedert worden. 
Reichskassenverwalter stellv. Reichskassenverwalter HAL

HAL

\section{Amt für Finanzverwaltung}

Amt für Rechtsverwaltung

HAL

HAL

$\mathrm{AL}$

Amt für Allgemeine Verwaltung ${ }^{16}$ AL

\section{Wirtschaftsamt}

12/1939 Verw.-Obf. Willy Heitmüller Gbf. Ludwig Grimm ${ }^{15}$

Hbf. Hans Dreblow

Bf. Kurt Arnold

Dr. Kurt Wohlfeil

Obf. Hellmuth Bayer

ab 1/1943 Obf. Walter Bergemann

Hgf. Dr. Kurt Wohlfeil

Ostf. Herbert Lindscheid

Obf. Werner Opdenhoff

Gbf. Ludwig Grimm Josef Stolz

\section{HJ-Dienststellen bei den Reichsvertretungen}

Bulgarien
Dänemark
Frankreich
Griechenland
Italien
Rumänien
Slowakei
Spanien
Ungarn

Bulgarien

änemark

Griechenland

Rumänien

Slowakei

Spanien
1940 Obf. Günther David, ab 8/1942 Obf. Hellmuth Bayer

\section{Auslandsbefehlsstellen der RJF ${ }^{17}$ \\ Befehlsstelle Böhmen und Mähren, Prag $^{18}$ \\ Befehlsstelle Generalgouvernement, Krakau ${ }^{19}$} Hbf. Theodor Stadler, Gbf. Günther Blum
Befehlsstelle Niederlande, Den $\mathrm{Haag}^{20}$ sowie danach, z.T. uberlappend: 6/1942 Obf. Wilhelm Voß, Gmf. Wilhelmine Hagemense, ab 11/1942 Gbf. Willi Blomquist, Gbf. Theodor Stadler

Obf Lutz Hassenpflug ab 11/1942 Hbf. Emil Teichmann. Hmf. Gerda Wend Obf. Heinz Schmidt, ab 6/1942 Obf. Hermann Theilen, bis 11/1942 Bf. Adolf Janßen

Ogff. Graf Oskar von Bothmer

Hbf. Heinz Wilke, Obf. Rolf Redeker Hbf. Günther Muths, ab 11/1942 Obf. Lutz Hassenpflug ab 2/1942 Obf. Heinz Förster, ab 11/1942 Hbf. Günther Muths bis $11 / 1942$ Hbf. Gerhard Bennewitz ab 2/1942 Obf. Friedrich Schumacher

Befehlsstelle Norwegen, Oslo

Befehlsstelle Belgien, Brüssel ${ }^{21}$
Gbf. Kurt Petter

1-4/1943 Bf. Hans Meßmacher, Obf. Erich Lemmer Hmf. Ursula Meßmacher, ab 8/1943 Bmf. Elis Feichtinger

Ostf. Hermann Degen, ab 1/1942 Obf. Rudolf Hemesath ab 6/1942 Bf. Adolf Janßen, ab 11/1942 Gbf. Gerhard Bennewitz

151943 vertreten durch Obf. Waiter Bergemann.

16 Das Amt für Allgemeine Verwaltung ist im Zuge "kriegsbedingter Kräfteeinsparungen" im Januar 1943 aufgelöst worden; seine Aufgaben wurden vom Wirtschaftsamt und vom Amt für Rechtsverwaltung wahrgenommen.

17 Die Außenbefehlsstellen trugen ab 3/1940 den "Charakter eines Amtes der Reichsjugendführung".

18 Mit den HJ-Bannen Brünn, Budweis, Iglau, Klattau-Pilsen, Königgrätz, Mährisch-Ostrau, Olmütz, Pilsen, Prag; ab 1/1943 wurde die Befehlsstelle Böhmen und Mähren während der Wehrmachtseinsätze von Hbf. Zoglmann zeitweise von Gbf. Friedrich Knoop geleitet.

19 Mit den HJ-Bannen Deutsch Przemysl, Krakau-Land, Krakau-Stadt, Lemberg, Lowitz-Zyrardow, Lublin, Neu-Sandez, Radom, Radomsko, Stanislau-Lemberg, Tomaschow, Warschau-Land, Warschau-Stadt.

20 Mit den HJ-Bannen Den Haag, Gelderland-Amheim, Groningen, Limburg-Maastricht, Nord-Brabant-Eindhoven, Nord-HollandAmsterdam, Overiyssel-Hengelo, Seeland-Rotterdam, Süd-Holland-Rotterdam, Utrecht.

21 Der Chef der Befehlsstelle Brüssel war zugleich Beauftragter des RJF beim Militär-Befehlshaber für Belgien und Nordfrankreich. 
Befehlsstelle Osten der RJF, Berlin

Befehlsstelle Ostland, Riga ${ }^{22}$

Befehlsstelle Ukraine, Rowno ${ }^{23}$
Hbf. Siegfried Nickel

Hmf. Ilse Stobbe, ab 1/1944 Gmf. Gertrud Eggemann

Gbf. Heinrich Lüer, Gmf. Ria Penke

Obf. Hinrich Petersen, Obf. Wilhelm Schulz, Hmf. Ilse Bluhm, Bf. Fritz Thiele Hbf. Georg Segel, Bf. Erich Bogun, Hmf. Ruth Jondral, Bf. Helmut Weinert

Gmf. Else Storck

\section{HJ-Landesjugendführungen ${ }^{24}$}

Afghanistan
Belgien
Bulgarien
China
Dänemark
Finnland
Frankreich
Griechenland
GroBbritannien
Italien
Japan
Kroatien
Norwegen
Portugal
Rumänien
Schweden
Schweiz
Serbien
Slowakei
Spanien
Türkei
Ungarn

Bf. Fritz Hoffmann, Bmf. Anne-Marie Schmidt Bf. Walter Schmidt, ab 10/1943 Ostf. Wilhelm Hardt Bf. Erich Popp, Liselotte Rubbe Gff. Helmut Wiedemann, Mrf. Margarete Pflug Obf. Lutz Hassenpflug 5/1943 - 9/1944 Bf. Hans Bäder, Rgf. Anna-Lisa Metzger Obf. Hermann Theilen, ab 12/1943 Hgf. Hubert Kiel, Bmf. Ilona Otten Werner Peek, Rgf. Maria Moseke Gff. Kurt-Dieter Czechanowski Bf. Götz Krack, ab 1 1/1942 Obf. Rolf Redeker

Gbf. Reinhold Schulze, danach Ogff. Heinrich Mielke Ogff. Wilhelm von Seiller, danach Gff. Albrecht, Stf. Herbert Oertel Ogff. Herbert Reiher, danach Obf. Erich Lemmer, Bmf. Elis Feichtinger

Stf. Dr. Kurt Schüppel, Mrf. Aenne Mertens Hbf. Günther Muths, ab 11/1942 Obf. Lutz Hassenpflug, Hmf. Marlis Hermann Ogff. Dr. Heinz Carsten Bf. Heinz Heinemann; nach 1942 Obf. Heinrich Bieg, Rgf. Hilde Bohnert Ogff. Dr. Josef. Dubitzky, ab 3/1943 Oschf. Johannes Kunze Obf. Richard Heil, ab 8/1944 Obf. Kurt Sandstede ab 7/1941 Gf. Käthe Oertel, ab 1/1944 Hmf. Hertha Bochmann Bf. Detlev Ehlers, Mrf. Erika Ludwig Bf. Herbert Rupf, Rgf. Oberender Gff. Heinz Schlicht, ab 5/1944 Gbf. Günther Prager

\section{Landesjugendführungen der "Deutschen Jugend" in Europa}

Banat
Dänemark
Kroatien
Rumänien
Slowakei
Ungarn

Hitler-Jugend-Einsatzstab in Litzmannstadt ${ }^{25}$

HJ-Vertretung bei der Wehrmacht ${ }^{26}$
LJF Franz German, LMF Luise Heinermann LJF Jef Blume, LMF Heie Erichsen

LJF Friedrich Haug, LMF Ursula Griesemann LJF Wilhelm Depner, LMF Melitta Baier LJF Ferdinand Klug, LMF Klara Scherer LJF Franz Huber, LMF Gisela Vogel

22 Mit den HJ-Bezirksstellen Estland-Reval, Lettland-Riga, Litauen-Kauen, Weißruthenien-Minsk

23 Mit den HJ-Bezirksstellen Wolhynien/Podolien-Luzk, Shitomir, Kiew, Dnjepropetrowsk und Nikolajew, sowie dem HJ-Aufbaustab in Tschernigow und der HJ-Landesführung Transnistrien.

24 Stand vom Januar 1943.

25 Dies war die zentrale HJ-Dienststelle beim Reichskommissar für die Festigung deutschen Volkstums und bei der Außenstelle des Rasse- und Siedlungshaupamtes der SS in Litzmannstadt.

26 Vertreter des Oberkommandos der Wehrmacht bei der Reichsjugendführung war bis 5/1940 Oberstleutnant Paul Völckers, danach Hauptmann Ernst Kaether. 


\section{Führer- und Führerinnenschulen der Reichsjugendführung ${ }^{27}$}

Akademie für Jugendführung Braunschweig

Gbf. Kurt Petter, ab 1942 Gbf. Kurt Budäus

BDM-Vertreterin

Gmf. Luise Michel

Reichsführerschule 1 Potsdam ${ }^{28}$

Reichsführerinnenschule 1 Potsdam

Reichsfuihrerinnenschule 2 Potsdam-Babelsberg

Reichsführerinnenschule 3 Hartenstein (für BDM-Werk "Glaube und Schönheit")

bis $8 / 1938$ Hbf. Paul Minke, bis 7/1939 Obf. Günther Bertelmann,

Gbf. Dr. Kurt Petter, ab 6/1942 Hbf. Thomas Stöckl

Gmf. Luise Michel, ab 7/194I Gmf. Hilde Meerkamp

Rmf. Ilse Behrens

Reichsführerinnenschule 4 Boyden

Gmf. Margret Klein, Rmf. Ilse Behrens, ab 1940/42 Hmf. Käthe Paulsen

Reichsführerinnenschule (5) Mittelburg/Eutin

\section{Fach-, Verwaltungs- und Sportschulen der Reichsjugendführung ${ }^{29}$}

Auslandsdeutsche Schülerheime, Stuttgart

Bf. Hermann Stockinger

B.u.L.-Stellenleiterinnenschule, Boyden/Ostpreußen

Fachschule für Jugendwohnheimleiter I, Braunschweig

Bf. Harald Mordhorst

Fachschule für Jugendwohnheimleiter II, Warschau

Obf. Reinhold Wedewen

Fachschule für Jugendwohnheimführerinnen, NeuschloßHohenmauth/Böhmen

Hmf. Lentz

Führerschule für Umsiedler, Litzmannstadt/Wartheland

Gymnastikschule des BDM-Werks, Stuttgart-Degerloch

Württemberg

KLV-Musikschule, Klein Desha/Sachsen

KLV-Schule 1 für Lagermädelführerinnen, Zell/Nürnberg, Franken

KLV-Schule 1 für Lagermannschaftsführer, Steinau/Oder

Hbf. Herbert Frehse

KLV-Schule 2 für Lagermädelschaftsführerinnen,

Harrachsdorf/Sudetenland

KLV-Werkschule 1, Prag

KLV-Werkschule 2, Ambach/Starnberger See

L-Stellenleiterinnenschule, Traunstein/Hochland

Osteinsatzschule, Hochsteinau/Oberschlesien

Ostlandführerschule, Marienwerder/Ordensschloß

Reichsausbildungslager 1, Heiligenstadt/Thüringen

Reichsausbildungslager 2, Germeter/Köln-Aachen

Reichsausbildungslager 3, Eningen/Reutlingen, Württem-berg

Reichsausbildungslager 4, Steegskopf/Westerwald, Hessen-Nassau

Reichsausbildungslager 5, Bistritz am Hostein

Reichsausbildungslager 6, Dessau/Mittelelbe

Reichsausbildungslager für Streifendienstführer, Freusburg/Sieg, Köln-Aachen

Reichsfunkschule, Berlin-Charlottenburg

Reichslanddienstführerschule 1, Groß Gurek/Skotschau, Oberschlesien

Reichslanddienstführerschule 2, Burgstadt/Wartheland Reichslanddienstführerinnenschule I, Wünschendorf/ Lauban, Niederschlesien

Reichslanddienstführerinnenschule 2, Mendischwetz/ Skotschau, Oberschlesien
Reichslanddienstlehrhof "Alpenland", Kuchlbach/Salzburg Reichslehrküche, Koppenmühl

Reichsnachrichtensportschule, Plauen/Sachsen

Reichsreiterschule, Plauen/Sachsen

Reichsschieß- und Geländeschule 1, Boyden/Ostpreußen

Reichsschieß- und Geländeschule 2, Potsdam/Mark Brandenburg

Reichsschieß- und Geländeschule 3, Mittelburg/Franken

Reichsschießschule, Obermaßfeld-Suhl/Thüringen

Reichsschule für soziale Jugendarbeit, Schloß Cammer bei Golzow/Mark Brandenburg Ostf. Siegfried Faßbender Reichsschule für weltanschauliche Schulung, Schloß Molchow/Neuruppin, Mark Brandenburg bis 7/1942 Obf. Albert Kilian

Reichsschulungsburg, Erwitte/Westfalen-Süd

Reichsseesportschule 1 "Gorch Fock", Prieros/Mark Brandenburg

Reichsseesportschule 2, Seemos-Bodensee/Baden

Reichsseesportschule 3 "Admiral von Trotha", Ziegenort, Kreis Ueckermünde/Pommern

Reichsseesportschule 4 "Admiral Wilhelm von Tegetthoff", Neusiedl am See/Wien

Reichsseesportschule 5, Eltville/Rhein, Hessen-Nassau Reichsseesportschule 6, Heisternest/Danzig-Westpreußen Reichssegelflugschule 1, Trebbin/Mark Brandenburg Reichssegelflugschule 2, Schwangau/Hochland Reichssportschule 1, Braunau/Bober, Kreis Löwenberg/ Niederschlesien

Reichssportschule 2, Freiburg/Breisgau, Baden Reichssportschule 3, Stuttgart-Degerloch, Württemberg Reichssportschule 4, Gladbeck/Niedersachsen Reichssportschule 5, St. Ruprecht/Villach, Kärnten Reichsversehrtenschule, Buckow/Mark Brandenburg Reichsverwaltungsführerschule, Niedernhausen/Taunus Reichsverwaltungsführerschule, Traunstein/Hochland Seminar für Musikerzieher der Hitlerjugend, Weimar Seminar für Musikerzieher der Hitlerjugend, Graz Seminar für Musikerzieher der Hitlerjugend, Berlin Volksdeutsche Führerinnenschule, Stuttgart Werkschule Bad Schandau-Ostrau Else Tümmel

27 Aufgeführt sind hier nur die zentralen, der Reichsjugendführung direkt unterstehenden Reichsführerschulen; daneben bestanden 1943 in den $42 \mathrm{HJ}$-Gebieten $71 \mathrm{HJ}$-Führer- und 52 BDM-Führerinnenschulen, die den jeweiligen HJ-Gebietsführungen direkt unterstanden, hinzu kamen in jedem Bann/Untergau mindestens eine Bannführer-/Untergauführerinnenschule. Im Krieg vor allem Lehrgänge für ausländische Jugendfuihrer.

29 Aufgeführt sind hier nur die zentralen, der Reichsjugendführung direkt unterstehenden Spezialschulen; hinzu kamen zahlreiche, den Gebiets-/Obergaufiihrungen unterstehende Schulungsstätten. 
HJ-Reichsbanne

Reichsbann Gehörgeschädigte (G) mit Sitz in Tilsit Reichsbann Blinde (B) mit Sitz in Stuttgart

Reichsbann Binnenschiffahrt (BS) mit Sitz in Berlin

Reichsbann Seeschiffahrt (S) mit Sitz in Berlin

\section{Zentrale HJ-Zeitungen}

Reichs-Jugend-Pressedienst

Junge Welt

Das Junge Deutschland

Das Deutsche Mädel

Der Pimpf

Wille und Macht
Hbf. Günter Kaufmann Hauptschriftleiter Bf. Wilhelm Utermann, ab 5/1942 Bf. Herbert Reinecker Hauptschriftleiter Bf. Albert Müller Hauptschriftleiterin Ogf. Hilde Munske Hauptschriftleiter Bf. Wilhelm Utermann, ab 5/1942 Bf. (1943 Obf.) Herbert Reinecker Hauptschriftleiter Gbf. Günter Kaufmann 